\title{
Institutional Policy for ALN
}

\author{
Dale A. Harris and Andy DiPaolo \\ Stanford University \\ Durand 307 \\ Stanford, CA 94305-9510 \\ Phone: (650) 725-0433 \\ E-mail: harris@Isl.stanford.edu, adp@ leland.Stanford.EDU
}

\begin{abstract}
For the past five years, Stanford has been involved in developing the capability to offer courses to remote learners over the Internet. This has evolved into a robust operation, which over the past year has offered 180 courses to approximately 4000 distance learners. More recently, the Department of Electrical Engineering has extended its offerings so that it is now possible to earn course credits sufficient to obtain a Master's degree and academic certificates entirely online. In this paper, we discuss the issues of institutional policy which have emerged as we have gone through this evolution. Our experience at Stanford will be discussed within the broader framework of institutional policy and of the general institutional resistance to change in higher education. A version of this paper was presented at the Fourth International Conference on Asynchronous Learning Networks held in New York in November 1998.
\end{abstract}

\section{KEYWORDS}

Institutional policy, Higher education, Online education

\section{ONLINE EDUCATION AT STANFORD}

\section{A. The Experimental or Trial Phase}

With financial support from the Sloan Foundation, Stanford began investigating the possibility of offering online courses in 1994 [1]. Stanford has a history, dating back to the 1970s, of offering classes to distance learners via broadcast television. The classes offered were almost exclusively graduate level engineering courses that could be taken for degree credit or for non-degree credit as appropriate. The targeted learners were practicing engineers, scientists, and technology managers. For Stanford, delivery of courses over the Internet was an extension of the existing broadcast television system. Classrooms equipped with cameras and microphones were already in place as was the administrative processes for marketing the courses and managing the distance learners. Our belief was that online delivery would first supplement and later possibly even replace broadcast television as a delivery mechanism. Our task was to determine if the technology to support online delivery was available and to integrate the appropriate systems to make it all work on an experimental or trial basis.

Since teaching distance learners for course credit was already established policy in the School of Engineering at Stanford, our major policy issue was one of faculty time. If placing a course online were to result in any additional time burdens on the instructor, we would face massive resistance regardless of how well intentioned the initiative was. This issue is a common one among universities considering development of an ALN or any kind of distance education program. Will policy be changed so that those instructors willing to become involved in development of a new teaching method will receive some sort of credit or compensation for doing so? What are the reward systems and motivations for faculty to engage in an activity of this type? For example, might they have their teaching load reduced in other areas? We did not feel that such a policy change was a realistic possibility. In response, we committed 
ourselves to the stealth approach; that is, to creating an online course delivery platform and process which would be essentially transparent to the instructor and could progress unnoticed as much as possible.

We accomplished this by developing a video-on-demand model for course delivery. We videotaped the broadcast lectures, digitized them, and made them available on-demand over the Internet as streamed video and audio. Other course materials, such as handouts and class notes, were also digitized and made available online. The students were allowed to take the courses asynchronously only up to a point. They did not have to be present or online at the time the lectures were given. However, their homework due dates and exams were synchronized with those for students taking the classes on-campus or by broadcast television. To the instructor, there was no difference between an Internet student and the traditional television student. There was the possibility that Internet delivery might increase the total number of students; however, for the purposes of the trial phase, we put that problem off into the future by limiting the Internet students to a very small number for any given course.

\section{B. The Stanford Online Operation}

We completed the trial phase with three key results. First, the technology was available to meet our goals. It was judged to be marginally satisfactory in some cases, but satisfactory nonetheless. And of course, we could also expect that situation to improve rapidly. Second, we learned that there was a market or user base for online delivery of Stanford courses. And third, we learned that at least when deployed on a small scale, it was possible to accommodate online students in a way that was functionally transparent to the faculty. In fact, some faculty found online delivery provided an unexpected bonus. Television students who venture out of range of our broadcast system (the San Francisco Bay Area) have traditionally been supplied with videotapes of lectures. The delays associated with mailing the tapes meant that the student was often behind and out of synchronization with the campus students. Such students had to be dealt with by the instructor on an exception basis. With online availability of lectures, no student had to be dealt with as an exception because no student was ever out of range of the Internet.

With these results in mind, Stanford Online was launched in 1997 with the financial support of the Sloan Foundation and equipment grants from Compaq and Microsoft. Stanford Online is a fully professionalized operation responsible for capturing, digitizing, and delivering online courses over the Internet [2]. Compared to the experimental and trial phase, Stanford Online is much larger scale and more robust in every way. During this academic year, over 100 courses will be offered to approximately 1000 students online. In addition to the video and audio of lectures, Stanford Online also utilizes a separate window for displaying the presentation graphics accompanying the lecture and an indexing scheme that allows access to parts of lectures by topic.

As Stanford Online began to scale up, Internet course delivery began to emerge from its stealthy cloak and became increasingly visible to those concerned with policy issues. Of particular interest was the decision to offer a complete Master's degree in electrical engineering online. The remainder of this paper discusses the relevant institutional policy issues, the varied approaches for dealing with them, and how we are approaching these policy issues at Stanford.

\section{RESISTANCE TO CHANGE}

David Jaffee [3] has argued that institutions of higher education are not run like businesses or even government bureaucracies. To varying degrees, these organizations are involved in "continual assessment, reflection, self-transformation, productivity gains, and quality improvement." On the other hand, institutions of higher education are social organizations characterized by "traditions, cultures, norms, and institutional missions." These are all reflected in the decision-making processes of the university, which places great power in the hands of the faculty and distributes the making of policy 
decisions across the full spectrum of organizational units. Policy is set by the university, by the school or college, by the academic department, and by the individual instructor. Worse yet, policy is often set at one level, interpreted at another level, and executed and monitored at a third. Some of the most staunchly defended policies are de facto, a result of custom and tradition rather than purposeful administration. It should come as no surprise that far reaching policy changes are hard to come by in the university.

\section{INSTITUTIONAL POLICY FOR ALN}

\section{A. Policy Around ALN Courses}

This is most often a policy of enablement by the administration with the final policy for a given course left to the individual instructor. The decision to allow credit for ALN courses most often rests with the school or college. In a small university, there could be a university-wide policy. Which courses will be made available by ALN is most often the decision of the academic departments in conjunction with the extension program if one exists. Academic departments are usually also responsible for determining policy regarding any incentive, additional compensation, or reduction in course load that might be offered to faculty who take on additional students through ALN. At smaller universities, these policy decisions might be made above the department level. The interpretation of policy and the final decision as to whether to teach via ALN is usually left to the individual faculty member. In effect, faculty members develop a series of individual policies regarding the teaching of an ALN course. These individual policies include not only whether they will teach via ALN, but also under what conditions they will agree to do so.

Another interesting policy issue is the use of ALN for campus students. At Stanford, this is primarily up to the course instructor. Some instructors embed use of ALN into their courses. One of us (Harris) routinely records course lectures onto an ALN server and requires all students, both on campus and beyond, to access the lectures online. Class time is then used for discussion. What about students on campus who want to take two courses offered in the same time slot? Should they be allowed to take one of them via ALN? At Stanford, this decision is left to the instructor of the course. Most faculty, but not all, will accommodate the occasional student who wants to do this.

\section{B. Policy Around ALN Degrees}

Decisions here revolve around whether to allow ALN course credits to be applied toward an academic degree, whether a degree can be obtained entirely by ALN (which requires dropping any residency requirements), and whether a degree obtained by ALN will be identical to or different from the degree obtained by traditional on-campus students.

The issue of degrees strikes at the core of academic institutional identity, both collectively and individually. Degrees are the currency by which universities are valued. Like any currency their value can fluctuate based on a large number of interacting factors. University policy around degrees is a particularly serious matter. Policy concerning undergraduate degrees usually rests with the university and with the school or college. Policy concerning graduate degrees usually rests with the school or college and with the academic departments. In our quest to offer an online Master's degree in electrical engineering at Stanford, we found that the decision to offer the degree and the decision to drop the existing residency requirement rested with the electrical engineering department. Some other departments in the School of Engineering had no current residency requirement for the Master's degree. In electrical engineering, there was an existing one-quarter residency requirement that could be waived by the department. Amid some controversy, we were successful with the desired policy change. At the same time, it was clear that if we wanted a broader ranging policy decision concerning degrees (other degrees and/or other departments), it would be a very complex and perhaps contentious matter. 


\section{Copyright Policy}

There is no reason to review this issue in depth here since it is much discussed elsewhere in the literature [4], [5]. Suffice it to say that we have updated our copyright policy at Stanford. There are now broad conditions under which the university will own the rights. These conditions include material developed using Stanford facilities and resources for a Stanford course. In short, in most cases, a Stanford professor cannot unilaterally market his or her course outside the university, whether done via ALN or otherwise. For video and/or audio recordings of what transpires in a Stanford classroom, Stanford has the first right of refusal for ownership. In particular, any electronic representation of the course, such as done by Stanford Online, belongs to the university. This policy update was made for a number of reasons, including the belief that the emerging technologies and widespread advent of online education renders the older policy out of date.

\section{Policy on Use of Faculty}

This includes a number of well-discussed issues including the use of part-time faculty, teaching load, and the use and future of tenure. All of these are issues with a life quite independent of ALN [6,7]. However, the growth of ALN brings additional urgency to the table. Like copyright policy, these are not problems which must be solved to enable ALN, rather they represent policies which may need to be reformed in reaction to ALN and the increasing use of educational technologies in general.

\section{E. Shifting Power Relationships}

The above discussion shows that the advent of ALN and its rapid growth raise a wide range of policy issues, some of which strike directly at the "traditions, cultures, norms, and institutional missions" by which universities are run. Shifts in power relationships within the institution are becoming clearly evident. For example, in most institutions, distance education has been treated as a necessary adjunct to the "real academic purpose" of the university, which has been to educate those matriculating students on campus. Distance education programs have been initiated for political reasons (community outreach) and for financial reasons (new revenue source). At Stanford, our distance education program has always been well integrated with the School of Engineering as a means to enhance relationships with industry. Our courses taught to distance learners have traditionally been the same courses as those taught to on-campus students. They have been offered by the same faculty. Among universities, Stanford has represented the exception rather than the rule. Most typically, distance education courses have been specifically designed for continuing learners and often taught by adjunct faculty. For the typical faculty member at a university, the distance education program has been out of sight and out of mind.

ALN is changing that. The profile of distance education has been raised, integration of distance education and on-campus course development is close at hand, and there is a growing view that educating students beyond the campus may become a major element of a university's mission [8]. In turn, policy decisions regarding education beyond the campus as well as the impact of ALN on the campus itself are gaining the attention of faculty and administrators who previously saw such decisions as non-issues or as minor diversions. Shifting power relationships are occurring. For example, in some cases, traditional academic departments are under increased pressure to cooperate, collaborate, and even integrate with the extension program. This can be viewed as an exciting event; but at the same time reaction, conflict, and inertia are also expected because of the nature of university governance.

\section{F. The Choices for Proponents of ALN}

It would seem that proponents of ALN have three choices. One approach is confrontational. Using this approach, proponents would design what they believe to be optimal ALN policies and push for their adoption. ALN proponents would embrace their growing power base and fight for turf within the structure of the university. 
A second approach is to fit in. Using this approach, proponents would attempt to shape ALN to adhere to existing policies. If policy changes are necessary, they are to be done making as few waves as possible.

The third approach is to become as independent of university policy as possible. The model for this approach is the independent subsidiary. ALN and distance learning activities and operations would be made independent of the normal governance procedures of the university. Such organizations have greater flexibility when compared to their internal counterparts. They would operate as a small entrepreneurial business. Several universities are taking this approach believing that education beyond the campus will become highly competitive. They believe that only an organization with the flexibility of an independent, customer-focused business will be able to take full advantage of emerging opportunities and to compete effectively with the growing number of for-profit, private businesses aimed at education and training beyond the traditional university campus [8].

\section{STANFORD'S APPROACH}

At Stanford, we are following the "fit-in" approach. As mentioned previously, Stanford has a long history with distance education. The organization responsible for distance education at Stanford (the Stanford Center for Professional Development) has been well integrated within the University and at least in the School of Engineering, faculty have been directly involved for many years in teaching at a distance via instructional television. Many policy and campus culture hurdles have long since been cleared. At the same time, there are those opposed to ALN and to further extension of Stanford beyond the campus. In spite of that resistance, at least to some extent Stanford has tradition, culture, and institutional mission working in favor of ALN. Our approach is to try to leverage that advantage.

How are we shaping ALN to adhere to current policies? We have already discussed our emphasis on making the technology and the ALN teaching process as transparent as possible for participating faculty. Regarding our online Master's degree, we are also committed to using the existing criteria and standards for student selection. Thus, students pursuing the degree online will be equally qualified to those studying on-campus. We are also committed to the use of existing course requirements for the degree. Thus, the online degree is identical to that obtained on campus. These commitments allow us to avoid having to challenge the status quo in several very important respects. We are not attempting to change the quality of students admitted to Stanford nor or we attempting to change what students are expected to accomplish in order to obtain a degree.

Another element of our approach is to deal with policy issues at the lowest possible level. We have followed this approach when dealing with issues such as reluctant faculty, copyright concerns, residency requirements, and the portfolio of courses to be offered online. In practice, this means we deal directly with the faculty as well as the administration. As discussed earlier, this is possible and effective because of Stanford's previous history and acceptance of teaching graduate level engineering classes via broadcast television. So far, most faculty and administrators see ALN as not much different than what Stanford has been doing for years. This does not mean that our ALN activities are resistance free. But for the most part, we are fortunate to be able to focus on how to raise the quality of ALN education, rather than on how to get started or on deciding if it is the right thing to be doing at all.

We are committed to evaluate the success of ALN using the same criteria for success as we do for oncampus education. Again, this approach allows us to fit in with current policy rather than challenging it. Certainly student grades are an important aspect of measuring success as is student and faculty satisfaction. In addition, we will be assessing the acceptance of ALN students and graduates for industrial jobs and for further academic work. The latter deserves more comment. Approximately one third of students who obtain a MS degree in electrical engineering at Stanford go on to earn a Ph.D., 
MBA, or law degree. It may be that our online students, being employed technical professionals, may have different inclinations toward further education than their on-campus counterparts. We don't know. What is important is what happens if they choose to pursue further graduate work. They should be at least as successful as on-campus students in their applications for further study and in their completion rates.

Lastly, Stanford is fortunate in the level of personal connection and professional ties that are maintained between the university and its graduates. It is our policy to foster and make use of these ties in a variety of ways. We consider it important that ALN students are made to feel the same connection to the university and we will evaluate our ALN program accordingly.

\section{CONCLUSION}

ALN provides a wide array of policy challenges to the traditional university. At the same time, the university is arguably not well positioned to respond to these challenges as readily as would a modern corporation or even a modern government bureaucracy. There are varied approaches to dealing with this dilemma including challenging the establishment, fitting in as best as possible, or withdrawing into a separate business-like organization run independently from the university.

Our approach at Stanford is premised by our long history with teaching and learning at a distance using broadcast television. ALN is viewed as a logical extension or next step of our instructional television network. Faculty do not find teaching online significantly different from teaching over television. Indeed, we have designed our ALN program to make sure faculty feel that way. We expect our online students who are taking courses for Stanford credit to have the same qualifications in academic background as our on-campus students. We expect them to take the same courses, graduate with the same degrees, and leave Stanford with the same qualifications for jobs and for further academic work. We also expect their personal connection to the university, both during their studies and after they graduate, to be the same as for traditional students. By shaping our ALN program in this way, we are building on our experience and tradition of distance education and avoiding any serious conflicts with the existing culture of the university including its customs and policies. When policy issues must be addressed, we attempt to address them at the faculty level if possible which keeps faculty involved with and supportive of the program.

\section{REFERENCES}

1. Harris, D. A. and DiPaolo, A., Advancing Asynchronous Distance Education Using High-Speed Networks; IEEE Transactions on Education vol. 39, pp. 444-450, 1996.

2. Stanford Online; http://stanford-online.stanford.edu/

3. Jaffee, D., Institutionalized Resistance to Asynchronous Learning Networks, JALN Vol. 2, Issue 2, pp. 21-32, 1998.

4. Guernsey, L. and Young, J. R., Professors and Universities Anticipate Disputes Over the Earnings From Distance Learning, The Chronicle of Higher Education, June 5, 1998.

5. American Association of University Professors Committee R on Government Relations, Report on Distance Learning, Academe, May/June, 1998.

6. Kolodny, A., Failing the Future: A Dean Looks at Higher Education in the Twenty-First Century, Duke University Press, Box 90660, Durham, N.C, 1998.

7. Leatherman, C., Growing Use of Part-Time Professors Prompts Debate and Calls for Action, The Chronicle of Higher Education, Oct. 10, 1997.

8. Harris, D. A., Online Distance Education in the United States, IEEE Communications Magazine, Vol. 37 No. $3,1999$. 